\title{
Cambio en las concepciones de los docentes sobre la argumentación y su desarrollo en clase de ciencias
}

\section{Teachers' change of conceptions on argumentation and its development in science class}

\author{
Francisco Javier Ruiz Ortega \\ Universidad de Caldas, Manizales, Colombia \\ francisco.ruiz@ucaldas.edu.co \\ Conxita Márquez Bargalló \\ Universidad Autónoma de Barcelona \\ Conxita.Marquez@uab.es \\ Óscar Eugenio Tamayo Alzate \\ Universidad de Caldas, Manizales, Colombia \\ oscar.tamayo@ucaldas.edu.co
}

RESUMEN • Este estudio muestra los cambios en las concepciones sobre los aspectos epistemológicos, conceptuales, didácticos y sobre la estructura argumentativa de cinco maestros de educación primaria que participaron en un proceso de reflexión crítica sobre la argumentación y su desarrollo en clase de ciencias. Con el fin de obtener información para su análisis e identificación de los cambios en los cuatro aspectos antes mencionados, se aplicó al inicio y final del proceso un cuestionario. Los resultados mostraron un cambio significativo en los componentes: epistemológico, conceptual y educativo, y no tanto en el estructural. Con dichos resultados se ratificó la importancia de ofrecer espacios de participación de los profesores para discutir, evaluar y proponer mecanismos de mejora en sus prácticas docentes en el aula.

PALABRAS CLAVE: concepciones docentes; enseñanza dialógica; estructura argumentativa.

SUMMARY - This study shows the changes in the conceptions of the epistemological, conceptual, educational and on the structure of the arguments built by five primary teachers who took part in a process of critical discussion on argumentation and development in science class. In order to identify the changes in the four aspects, a content analysis of a questionnaire applied at the beginning and at the end of the process was done. The results showed a significant change in the epistemological, conceptual and educational components; and not that much in the structural one. Apart from that, the findings ratified the importance of offering spaces of participation for the teachers to discuss, evaluate and propose mechanisms to improve their practices in the classroom.

KEYWORDS: argumentation; teachers' conceptions; dialogic teaching; argumentative structure.

Fecha de recepción: julio 2012 • Aceptado: noviembre 2012

Ruiz Ortega, F., Márquez, C., Tamayo O. E.(2014) Cambio en las concepciones de los docentes sobre la argumentación y su desarrollo en clase de ciencias. Enseñanza de las 


\section{INTRODUCCIÓN}

Desde la lingüística y la epistemología actual, se reconoce que es en el razonamiento y la construcción de la ciencia donde la argumentación desempeña un papel central al servir de mediadora para la construcción de las relaciones sustantivas entre los modelos y las evidencias (Von Aufschnaiter, Erduran, Osborne y Simon, 2004).

Además, la argumentación como competencia científica es la actividad cognitiva y social que permite relacionar fenómenos, modelos, evidencias y explicaciones (Revel, Couló, Sibel, Furman, Iglesia y Adúriz-Bravo, 2005). De ahí que desarrollar la argumentación en el aula es facilitar, en primer lugar, la comprensión de los conceptos científicos al involucrar cada vez más al estudiante en el uso de instrumentos conceptuales y procedimentales y, en segundo lugar, comprender la racionalidad de la misma ciencia (Sardá y Sanmartí, 2000).

Asumir que uno de los fines de la investigación científica es la generación y justificación de enunciados y acciones encaminados a la comprensión de la naturaleza es aceptar que en la enseñanza de las ciencias se debe dar la oportunidad de desarrollar la capacidad de razonar y argumentar (JiménezAleixandre y Díaz, 2003), y para ello será necesario persuadir al estudiante y cambiar la autoridad centrada en el maestro o en el libro de texto para dejar paso a espacios dialógicos en los que se permita la construcción y discusión de argumentos científicos (Driver, Newton y Osborne, 1998).

Desde esta perspectiva, la investigación que se presenta persigue tres objetivos. En primer lugar, identificar las concepciones de cinco docentes de la educación primaria, de una institución oficial de la ciudad de Manizales (Colombia), sobre tres aspectos relacionados con el desarrollo de la argumentación en clase de ciencias. Estos aspectos son: a) epistemológico, donde interesa identificar qué relación establecen los docentes entre la argumentación y la construcción de la ciencia; $b$ ) conceptual, donde interesa conocer qué concepto tienen los docentes sobre la argumentación en clase de ciencias, y c) didáctico, que se orienta a identificar qué criterios tienen en cuenta los docentes para el desarrollo de la argumentación en clase de ciencias y cómo gestionan el aula. En segundo lugar, esta investigación busca identificar un cuarto aspecto en los docentes, el estructural, relacionado con la estructura de sus argumentos. Y, en tercer lugar, intenta mostrar el cambio en los aspectos antes mencionados, tras participar en un proceso de reflexión crítica sobre la argumentación y su desarrollo en el aula de ciencias.

Esta investigación forma parte de una más amplia que se viene desarrollando desde el año 2009 y en la cual se procura identificar, caracterizar y comprender cómo evolucionan los modelos de enseñanza en el desarrollo de la argumentación en clase de ciencias de dos de los cinco docentes que participan en el proceso de reflexión crítica. Una investigación que seguramente ayudará a la comprensión del pensamiento y el desempeño de los docentes y proporcionará herramientas para afrontar no solo las exigencias expresadas en la política educativa nacional o internacional, sino también para intentar formar ciudadanos y ciudadanas que puedan participar de manera informada y responsable en una sociedad cada vez más compleja.

\section{¿Por qué indagar las concepciones de los docentes sobre los aspectos epistemológico, con- ceptual y didáctico?}

El estudio de las concepciones de los docentes cuenta con una tradición de más de tres décadas, cuando se produjo el auge e importancia de las teorías implícitas, presaberes o preconcepciones. En la actualidad se siguen desarrollando trabajos alrededor del pensamiento docente que ratifican la necesidad de continuar investigando la formación docente y el ejercicio profesional (Schön, 1998; Gunstone et al., 1993; Mellado, Ruiz y Blanco, 1997; Reyes, Enrique y Perafán, 1997; Kaufman y Fumagalli, 2000; 
Hernández, 2000; Angulo, 2002; Fernández, Gil y Carrascosa, 2002; Marcelo, 2005; Fernández et al., 2009; Benarroch y Marín, 2011).

Algunos autores (Llinares, 1996; Moreno y Azcárate, 2003; Gil y Rico, 2003) manifiestan que hablar de concepciones de los docentes es referirse a "marcos organizadores, que tienen naturaleza cognitiva, y que condicionan la forma en que afrontamos las tareas" (Gil y Rico, 2003: 28). Además, según Moreno y Azcárate (2003), en el interior de las concepciones se pueden encontrar "creencias, significados, conceptos, proposiciones, reglas, imágenes mentales, preferencias, etc., que influyen en lo que se percibe y en los procesos de razonamiento que se realizan" (p. 267); argumentos que apoyan la importancia de analizar y comprender las concepciones de los docentes, pues estas afectan a la toma de decisiones en los procesos de planeación y ejecución de una propuesta de enseñanza.

Desde la investigación en este ámbito se ha ido consolidando un cuerpo teórico útil para comprender no solo las complejas relaciones que se dan entre el pensamiento del docente y su desempeño en el aula de clase, sino también para orientar propuestas de intervención que involucren a estos docentes en la comprensión y regulación de las relaciones entre su pensamiento y su desempeño en el aula. En este último aspecto encontramos algunos trabajos (Sutton, 1997; Driver, Newton y Osborne, 1998; Simon, Erduran y Osborne, 2006) en los que se evidencia la necesidad no solo de enseńar a argumentar, sino también de involucrar al docente para que tome conciencia y analice sus concepciones alrededor de la argumentación.

Manifestamos anteriormente que esta investigación forma parte de una más general en la que interesa identificar modelos de enseñanza y, para lograrlo, consideramos que dicha identificación exige conocer las concepciones de los docentes en, al menos, tres aspectos: el epistemológico, el didáctico y el conceptual. A continuación presentamos una justificación más detallada para cada uno de estos aspectos:

\section{Aspecto epistemológico}

Varios elementos soportan el estudio del componente epistemológico en la enseñanza de las ciencias, el primero de ellos lo retomamos de los planteamientos de Duschl (1998), para quien existe la necesidad de establecer objetivos epistemológicos que permitan evaluar los enunciados científicos; es decir, contextos epistémicos que se articulen a los conceptuales y socioculturales y ayuden, de esta manera, a la comprensión de las evidencias como herramientas de explicación y soporte de las afirmaciones o argumentaciones que manifiestan las comunidades científicas. El segundo elemento que apoya la importancia de este aspecto se relaciona con la ciencia cognitiva de Giere (1988), para quien la ciencia es una actividad cognitiva de producción de conocimiento, producción que pasa por la construcción de representaciones internas que los sujetos elaboran cuando utilizan sus capacidades cognitivas (memoria, lenguaje, percepción, imaginación) para interaccionar con el mundo. Desde esta perspectiva, interesa en la comprensión de la construcción de la ciencia encontrar explicaciones causales de lo que sucede en los individuos -y con sus interacciones- al construir la ciencia.

De igual manera, existen trabajos (Lemke, 1997; Sutton, 1997 y 2003; Driver, Newton y Osborne, 1998; Sanmartí, 2003; Jiménez-Aleixandre y Díaz, 2003; Erduran, Simon y Osborne, 2004; Izquierdo, 2006) que muestran claramente la importancia de establecer relaciones entre argumentación y el avance de la ciencia en los procesos de enseñanza y aprendizaje de las ciencias.

En esta investigación interesó identificar si los docentes reconocen la argumentación como un proceso de negociación y construcción de conocimiento científico y de la ciencia escolar. 


\section{Aspecto conceptual}

En cuanto al concepto de la argumentación en clases de ciencias, hemos identificado dos grandes perspectivas. La primera, cuyo mayor representante es Toulmin (1969), asume la argumentación como un acto de comunicación de datos, afirmaciones y justificaciones; aquí, más que los procesos de interacción social y la importancia de los contextos donde suceden los debates, interesa la estructura clara y precisa de los componentes de los argumentos. En el segundo grupo encontramos a autores (Sanmartí, 2003; Sutton, 2003; Erduran, Simon y Osborne, 2004; Jiménez-Aleixandre y Díaz, 2003, Larraín, 2007. Henao y Stipcich, 2008) que ven la argumentación desde una perspectiva funcional, es decir, como proceso social y dialógico de presentación de evidencias, afectado indiscutiblemente por los modelos mentales de los sujetos que se implican en los debates, por el contexto en el cual estos suceden y por la finalidad que se persigue con la presentación y justificación de las evidencias.

En esta investigación interesó conocer cuál o cuáles son las concepciones que tienen los docentes de la educación primaria sobre la argumentación en clase de ciencias y saber hacia qué perspectiva se orientan estas concepciones.

\section{Aspecto didáctico}

Numerosas investigaciones realizadas en torno a los componentes de la didáctica de las ciencias (Porlán, Rivero y Martín, 1997-1998; Caicedo, 1999; Hernández, 2000; Kaufman y Fumagalli, 2000) fomentan la necesidad de investigar no solo los aspectos que rodean al educando (motivación, intereses, expectativas y valores), sino también aquellos relacionados con el docente, como sus modelos conceptuales y epistemológicos sobre cómo conciben la ciencia, cómo la enseñan y cómo creen que se aprende.

También se identifican investigaciones desarrolladas alrededor de la argumentación en clase de ciencias (Sanmartí, 1997; Driver, Newton y Osborne, 1998; Zohar y Nemet, 2002; Jiménez-Aleixandre y Díaz, 2003; Campaner y De Longhi, 2005; Revel et al., 2005; Bravo, Puig y Jiménez-Aleixandre, 2009; Simon, Erduran y Osborne, 2006; Jiménez-Aleixandre y Erduran, 2008), que corroboran la necesidad de valorar la argumentación como "tarea epistémica y un proceso discursivo por excelencia en las ciencias" (Henao y Stipcich, 2008: 49). En este sentido, es la argumentación una competencia que hay que desarrollar en el aula de clase, no solo para promover una ciencia producto de la actividad humana sino también para potenciar el desarrollo de pensamiento crítico y de competencias ciudadanas que ayuden a la formación de las y los estudiantes.

Esta investigación tiene en cuenta, además de los trabajos presentados anteriormente, los supuestos planteados por Jiménez-Aleixandre (2010). Para ella, si se pretende mejorar la competencia de aprender a aprender, desarrollar el pensamiento crítico en las y los estudiantes y generar una apropiación de la cultura científica, es indispensable que en el interior del aula de clase se regulen y controlen los conocimientos, se den espacios permanentes para explicitar y analizar los procesos cognitivos que desarrollan las y los estudiantes, y aquí la argumentación desempeña un papel prioritario. Lograr lo anterior es facilitar, en primer lugar, que alumnas y alumnos hagan públicos sus razonamientos y procesos de pensamiento; en segundo lugar, que de manera informada y responsable tomen decisiones a favor de una transformación del aula de clase, y, en tercer lugar, que se conozca la estructura interna de la ciencia y se genere el desarrollo de prácticas propias del trabajo científico.

En la investigación realizada interesó conocer cómo el grupo de docentes promueven en el aula el desarrollo de la argumentación, qué criterios y actividades tienen en cuenta para lograr esta finalidad. 


\section{Aspecto estructural}

La estructura de un argumento ha sido uno de los aspectos que, desde la teoría argumentativa de Toulmin (1969), se ha incorporado en el campo de la enseńanza de la argumentación, al intentar explicitar la enseñanza de, al menos, tres de los elementos que constituyen el argumento: dato, afirmación y justificación.

Existen varias investigaciones cuyos objetivos centrales se han orientado no solo a identificar elementos que componen la estructura argumentativa de los textos orales o escritos elaborados por los estudiantes en clase de ciencias, sino también a promover de manera consciente el proceso de construcción de los argumentos (Dolz, 1995; Sanmartí, 1997; Erduran, Simon y Osborne, 2004; Hoffman, 2008; Henao y Stipcich, 2008; Erduran y Villamanan, 2009; Schwarz, Reiser, Davis, Kenyon, Achér, Fortus et al. 2009). También encontramos propuestas como la de Osborne, Erduran y Simon (2004), cuya intencionalidad es involucrar a los docentes no solo para que sean sujetos activos en la elaboración de propuestas didácticas proyectadas a la enseñanza de la argumentación en clase de ciencias, sino también para que sean sujetos reflexivos y críticos que construyen y evalúan sus propios argumentos.

Es innegable que, si se pretende que los docentes enseñen a argumentar a sus estudiantes en clase de ciencias, debemos ofrecer espacios dialógicos en donde ellos puedan, desde sus vivencias, experiencias y conocimientos, reflexionar y regular su propia forma de elaboración y evaluación de los argumentos. De ahí que en nuestra investigación se da importancia a involucrar a los docentes en el proceso de reflexión y crítica de su desempeño en el aula.

\section{OBJETIVO DE INVESTIGACIÓN}

Identificar los cambios en los aspectos epistemológico, conceptual, didáctico y estructural de cinco docentes de educación primaria, después de participar en un proceso de reflexión crítica sobre la argumentación y su desarrollo en el aula de ciencias.

\section{DISEÑO DE LA INVESTIGACIÓN}

\section{Contexto de la investigación}

La investigación se desarrolló con cinco docentes de educación primaria de una institución pública de la ciudad de Manizales (Colombia), llamada Fe y Alegría. Esta institución cuenta con aproximadamente 2.250 estudiantes y está ubicada en una zona marginal de la ciudad. Cuatro profesores son egresados de las Escuelas Normales, donde el énfasis principal es la pedagogía, y otra es egresada de una institución académica en la cual se permite, según los intereses y capacidades del estudiante, profundizar en un campo específico de las ciencias, las artes o las humanidades y acceder a la educación superior (Ministerio de Educación, Ley General 115, 1994). Los docentes orientan sus clases a niños y niñas cuyas edades están entre los 9 y 10 años. 


\section{Intervención y obtención de la información}

Para lograr el objetivo planteado anteriormente, se desarrollaron las siguientes actividades:

a) Aplicación de cuestionario. Se aplicó el mismo cuestionario al inicio y al final del proceso (siete meses después de comenzar el proceso de reflexión crítica).

b) Grabación de clases. Se visitó al grupo de docentes (cuatro de ellos) en el aula y se registró una clase antes de comenzar el proceso de reflexión crítica.

c) Realización de los tres primeros encuentros de reflexión crítica. Se realizaron, inicialmente, tres encuentros, donde a partir del material elaborado por el equipo de investigación se discutió la argumentación como competencia que desarrollar en el aula y se analizaron fragmentos de las clases registradas. Finalmente, se propuso al grupo de docentes diseñar una actividad con el objetivo de llevar a cabo la argumentación.

d) Grabación de clases. Se visitó, por segunda vez, a los cuatro docentes en el aula y se registró la segunda clase.

e) Realización del cuarto encuentro. Los participantes observaron, evaluaron y autoevaluaron los vídeos y, después, discutieron sus evaluaciones y autoevaluaciones con todos los integrantes del grupo en un cuarto encuentro programado para la reflexión crítica. Se propuso nuevamente al grupo de docentes el diseño de una segunda actividad para el desarrollo de la argumentación

f) Grabación de clases. Se visitó, por tercera vez, a los docentes y se registró la tercera clase.

g) Realización del quinto encuentro y aplicación por segunda vez del cuestionario. Esta actividad se desarrolló siete meses después de haberse iniciado el proceso.

h) Ejecución de entrevistas. En este caso se realizó una entrevista semiestructurada después de cada clase realizada por los docentes para reflexionar sobre su propio desempeño.

\section{Cuestionario}

El cuestionario está constituido por seis preguntas abiertas y cada una de ellas se relacionó con uno o más de los aspectos indagados (tabla 1).

Tabla 1.

Cuestionario aplicado antes y después del proceso de reflexión crítica

\begin{tabular}{|c|c|c|}
\hline Preguntas & Objetivos & $\begin{array}{c}\text { Aspectos } \\
\text { investigados }\end{array}$ \\
\hline $\begin{array}{l}\text { Existen dos ideas generalizadas de lo que es la ciencia: } \\
\text { a) La ciencia es un cúmulo de conocimientos objetivos y } \\
\text { absolutos, resultado de un proceso lineal que va de la } \\
\text { observación y experimentación sobre el mundo real a } \\
\text { las teorías científicas. } \\
\text { b) La ciencia es el resultado de un proceso de negociación } \\
\text { entre los integrantes de las comunidades científicas, en } \\
\text { donde se presentan, discuten y validan los modelos y } \\
\text { teorías construidos como representación del mundo. } \\
\text { 1. ¿Crees que los dos procesos son igual de importantes } \\
\text { para la construcción de la ciencia? Justifica tu respuesta. } \\
\text { 2. ¿Cuál de las dos ideas anteriores crees que es la que de- } \\
\text { bería orientar los procesos de enseñanza y aprendizaje de } \\
\text { las ciencias en el aula de clase? Justifica tu respuesta. }\end{array}$ & $\begin{array}{l}\text { Identificar si los docentes valoran la impli- } \\
\text { cación de la argumentación en los procesos } \\
\text { de construcción de las ciencias. } \\
\text { Identificar la estructura de sus argumentos. }\end{array}$ & $\begin{array}{c}\text { Epistemológico } \\
\text { Estructural }\end{array}$ \\
\hline
\end{tabular}




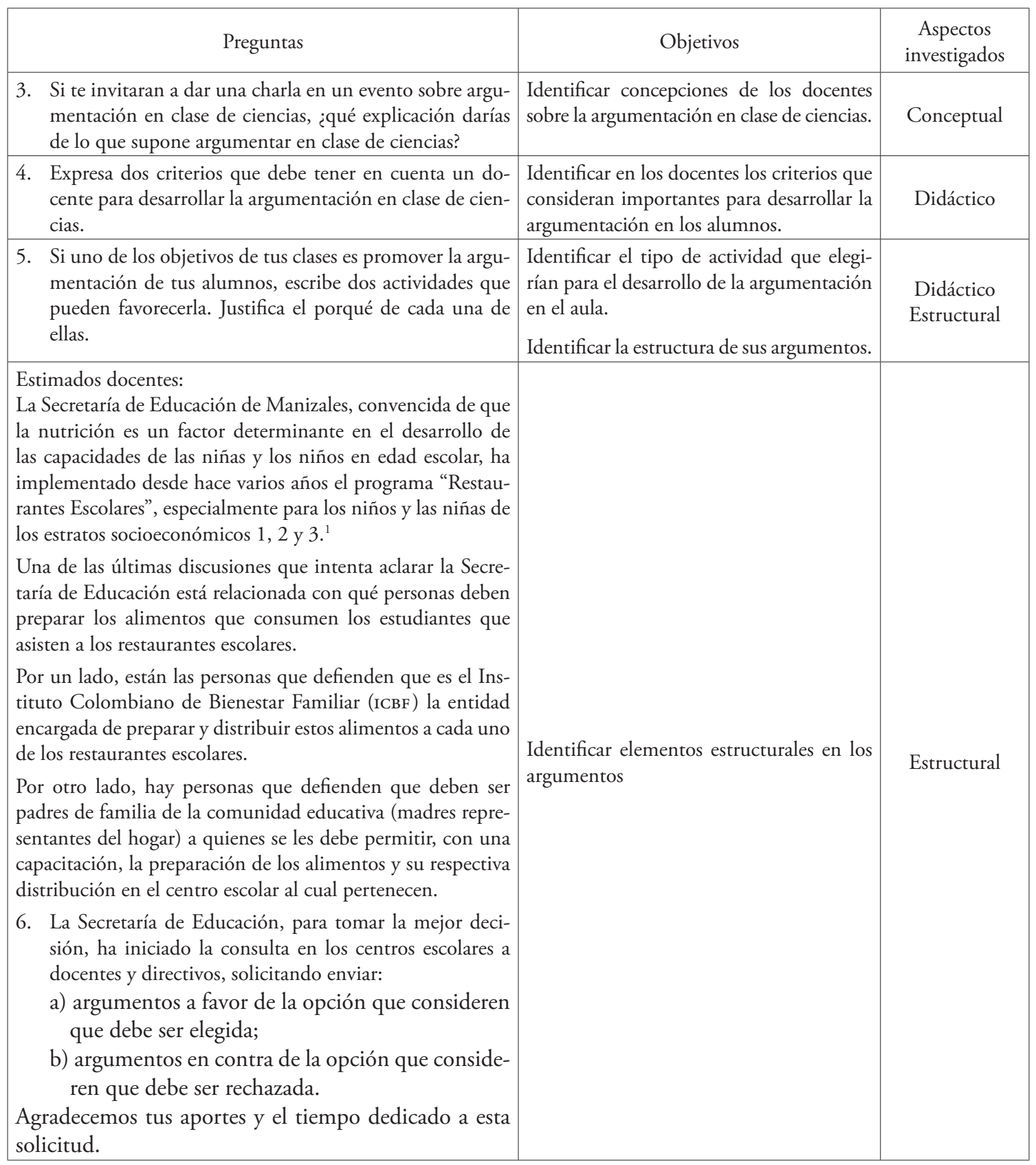

\section{Técnica de análisis de resultados}

Para caracterizar e identificar los cambios en los aspectos indagados se aplicó, tanto al cuestionario inicial como al cuestionario final, un análisis de contenido, en el que, a partir de los textos construidos, se infirieron elementos que permitieron caracterizar y justificar, dentro del contexto de la enseńanza y desarrollo de la argumentación en clase de ciencias, los cuatro aspectos estudiados.

1. Los estratos socioeconómicos en Colombia son una herramienta que utiliza el Estado para analizar a la población según aspectos como la dotación de servicios públicos domiciliarios, la ubicación de su vivienda (urbana, rural) y el salario, entre otros. En el país existen seis niveles o estratos socioeconómicos, siendo el de menores condiciones el estrato 1. 
La transcripción y codificación de la información se hizo con la ayuda del software Atlas-ti, y para concretar el análisis de contenido y la posterior cuantificación de los códigos construidos se aplicó una fórmula que permitió la elaboración de tablas de frecuencia: \% código $=\mathrm{n}{ }^{\circ}$ de citas del código x 100 / total de citas.

\section{RESULTADOS Y DISCUSIÓN}

A continuación se presenta, para cada uno de los aspectos indagados, la discusión de los resultados más relevantes obtenidos en la investigación.

\section{Aspecto epistemológico}

En la tabla 2 se muestran los resultados obtenidos para el aspecto epistemológico tras las dos aplicaciones del cuestionario:

Tabla 2.

Resultados obtenidos para el aspecto epistemológico en la aplicación inicial y final del cuestionario

\begin{tabular}{|c|c|c|c|c|}
\hline \multirow{2}{*}{ Aspecto } & \multicolumn{1}{|c|}{ Categoría } & Subcategoría & \multicolumn{2}{|c|}{ Resultados cuestionario } \\
\cline { 3 - 5 } & \multicolumn{1}{|c|}{$\begin{array}{l}\text { Inicial (100\%) } \\
\text { Epistemológico }\end{array}$} & $\begin{array}{l}\text { Relación entre argumenta- } \\
\text { ción y ciencia }\end{array}$ & 69,23 & 92,31 \\
\cline { 3 - 5 } & No & 30,77 & 7,69 \\
\hline
\end{tabular}

El grupo de docentes, ante las preguntas 1 y 2 planteadas para identificar este aspecto, manifestó, en la primera aplicación del cuestionario, su reconocimiento de la relación argumentación y ciencia. La evidencia de este reconocimiento alcanzó el 69,23\% del total de las respuestas obtenidas. En la segunda aplicación este porcentaje aumentó notablemente, obteniéndose un 92,31\% de reconocimiento de dicha relación.

Una de las respuestas que refleja el reconocimiento de la relación argumentación-ciencia obtenida en la primera aplicación del cuestionario fue la siguiente:

Pregunta: $1:$ ¿Crees que los dos procesos son igual de importantes para la construcción de la ciencia? Justifica tu respuesta.

Docente: Los dos procesos son importantes para la construcción de la ciencia porque en el proceso de la enseñanza de la ciencia debe existir la experimentación, la observación, la comparación, el análisis, la comprensión y el diálogo con otras personas que hacen el mismo experimento e investigación y llegar así a unas conclusiones.

Como se observa en la respuesta del docente, para él ambos procesos son importantes en la construcción del conocimiento científico. La justificación que da a su afirmación es que para llegar a una conclusión se requiere experimentar, observar, comprender e interactuar con otras personas. También añade a esta justificación que la comunidad o personas tienen que estar involucrados en el experimento o una experiencia de investigación. Sin embargo, esto no siempre ocurre en el proceso de construcción de la ciencia. 
En la segunda aplicación del cuestionario, el siguiente texto construido por uno de los docentes evidencia el reconocimiento de la relación entre argumentación y construcción de la ciencia:

Pregunta 1: ¿Crees que los dos procesos.....?

Docente: Sí, ya que el integrante de la comunidad científica primero tuvo que observar y experimentar para luego llegar a la presentación, discusión y validación en equipo.

El docente, además de dar importancia a los dos procesos, también reconoce que el debate y el trabajo en equipo son acciones relevantes para validar el conocimiento. Planteamiento que bien podríamos relacionarlo con aquellas propuestas teóricas en las cuales se asume que los argumentos discutidos, contrastados y validados en las comunidades científicas son herramientas útiles para la promoción y el desarrollo del conocimiento científico (Von Aufschnaiter, Erduran, Osborne y Simon, 2008). Además, se puede decir que esta respuesta se acerca también a las tendencias actuales que ven la argumentación como un mecanismo de sostenimiento de la calidad del conocimiento científico y como el escenario que permite la movilidad, desde un plano intrapsicológico del sujeto, hacia el plano interpsicológico, en el que ya la argumentación se convierte en una actividad dialógica (Erduran, Simon y Osborne, 2004).

\section{Aspecto conceptual}

En la tabla 3 se indican los resultados obtenidos para el aspecto conceptual:

Tabla 3.

Resultados obtenidos para el aspecto conceptual en la aplicación inicial y final del cuestionario

\begin{tabular}{|c|c|c|c|c|}
\hline \multirow{2}{*}{ Aspecto } & \multirow{2}{*}{ Categoría } & \multirow{2}{*}{ Subcategoría } & \multicolumn{2}{|c|}{ Resultados cuestionario } \\
\hline & & & Inicial $(100 \%)$ & Final $(100 \%)$ \\
\hline \multirow{3}{*}{ Conceptual } & Perspectiva estructural & La argumentación es un proceso informativo & 80 & 0 \\
\hline & \multirow[b]{2}{*}{ Perspectiva funcional } & La argumentación es un proceso dialógico & 20 & 42,9 \\
\hline & & $\begin{array}{l}\text { La argumentación es una herramienta para } \\
\text { evaluar el aprendizaje }\end{array}$ & 0 & 57,1 \\
\hline
\end{tabular}

La tercera pregunta planteada en el cuestionario (tabla 1) se orientó a indagar en las concepciones de los docentes sobre lo que supone argumentar en clase de ciencias. Ante la pregunta, el $80 \%$ de los textos construidos por los docentes en el cuestionario inicial se acercan a la perspectiva estructural de la argumentación (tabla 3). Desde esta perspectiva, argumentar es un proceso comunicativo de presentación de datos y afirmaciones cuya finalidad es informar o informarse. En la segunda aplicación, esta perspectiva ya no aparece. También se identificó, en el cuestionario inicial, la concepción de la argumentación como proceso dialógico ( $20 \%$ de los textos se ubicaron en esta perspectiva). Aquí, la argumentación es un proceso social. Fue satisfactorio encontrar que el porcentaje de textos ubicados en esta perspectiva aumentó en la aplicación del segundo cuestionario (con un 42,9\%). Por último, se resalta en los resultados del cuestionario final el surgimiento de un nuevo concepto de la argumentación, el hecho de asumirla como una herramienta útil para evaluar el aprendizaje de los estudiantes (con un $57,1 \%$ de frecuencia).

El texto siguiente, obtenido en la primera aplicación del cuestionario, muestra la perspectiva estructural de la argumentación, en la cual interesa, más que la interacción dialógica entre sujetos, la presentación clara de elementos constituyentes de los argumentos, como las afirmaciones o pruebas: 
Pregunta 3: Si te invitaran a dar una charla en un evento sobre argumentación en clase de ciencias, ¿qué explicación darías de lo que supone argumentar en clase de ciencias?

Docente: Argumentar es ofrecer un conjunto de razones o de pruebas en apoyo de una conclusión o de ciertas opiniones. A través del argumento tratar de informarse acerca de qué opiniones son mejores que otras.

En el cuestionario final, uno de los textos que evidencia la perspectiva funcional que concibe la argumentación como actividad social y dialógica fue el siguiente:

Pregunta 3: Si te invitaran a dar una charla en un evento sobre argumentación...

Docente: Argumentar en clase de ciencias es expresar lo que comprenden desde su propia cotidianidad, refutar conceptos si es necesario, decir el porqué de las cosas y explicar generando un ambiente de socialización donde se pueda debatir.

De manera similar, se identificó en el cuestionario final la aparición de una nueva concepción: la argumentación como herramienta para evaluar el aprendizaje, es decir, como mecanismo que potencia el desarrollo del pensamiento y la construcción conjunta del conocimiento escolar (Larraín, 2007). La siguiente respuesta es un ejemplo de esta concepción de la argumentación:

Pregunta 4: Si te invitaran a dar una charla en un evento sobre argumentación...

Docente: Argumentar en ciencias es una herramienta de la que disponemos para evaluar el conocimiento, es un proceso social que está afectado por ideas, prejuicios e instancias, potencializa cambios en las concepciones de los individuos, llevándolos a replantearse su saber, el de su entorno.

\section{Aspecto didáctico}

En el aspecto didáctico, fueron dos los elementos que se indagaron con las preguntas 4 y 5 del cuestionario. El primero, los criterios que el grupo de docentes considera que hay que tener en cuenta para el desarrollo de la argumentación. El segundo, las actividades de carácter grupal o individual que facilitarían también la obtención de este objetivo. En la tabla 4 se indican los resultados para este aspecto.

Tabla 4.

Resultados obtenidos para el aspecto didáctico en la aplicación inicial y final del cuestionario

\begin{tabular}{|c|c|c|c|c|}
\hline \multirow{2}{*}{ Aspecto } & \multirow{2}{*}{ Categoría } & \multirow{2}{*}{ Subcategoría } & \multicolumn{2}{|c|}{ Resultados cuestionario } \\
\hline & & & Inicial $(100 \%)$ & Final $(100 \%)$ \\
\hline \multirow{7}{*}{ Didáctico } & \multirow{5}{*}{$\begin{array}{l}\text { Criterios implicados en el desarrollo de la } \\
\text { argumentación: Estudiante (E), } \\
\text { Docente (D), } \\
\text { Saber }(S) \text {, } \\
\text { Contexto (C) }\end{array}$} & E & 22,22 & 0 \\
\hline & & E-D & 44,44 & 27,27 \\
\hline & & D-S & 33,33 & 0 \\
\hline & & E-D-S & 0 & 45,45 \\
\hline & & E-D-S-C & 0 & 27,27 \\
\hline & \multirow{2}{*}{ Gestión del aula: tipo de actividades } & Individuales & 50,00 & 10,00 \\
\hline & & Grupales & 50,00 & 90,00 \\
\hline
\end{tabular}


Analizaremos a continuación cada uno de los elementos indagados para el aspecto didáctico.

\section{Los criterios implicados en el desarrollo de la argumentación}

En relación con los criterios, es importante precisar que estos pueden centrarse en $a$ ) los estudiantes, sus habilidades cognitivas, actitudinales o procedimentales; $b$ ) los docentes, sus saberes y formas de enseñanza; $c$ ) el saber que se enseña; $d$ ) el contexto de los estudiantes, es decir, su cotidianidad, su mundo inmediato, y e) una combinación de dos o más de estos criterios.

En la tabla 4 se muestra que en la primera aplicación del cuestionario los criterios considerados por el grupo de docentes, necesarios para el desarrollo de la argumentación en clase de ciencias, estuvieron centrados en la relación estudiante-docente $(44,44 \%)$, luego ubicamos la relación docente-saber $(33,33 \%)$ y, por último, criterios que solo hacen referencia al estudiante $(22,22 \%)$. En el cuestionario final, se mantuvo como criterio, aunque en un porcentaje inferior a aquel obtenido en el primer cuestionario, la relación estudiante-docente $(27,27 \%)$, y emergen dos nuevos criterios: el primero, la relación estudiante-docente y saber $(45,45 \%)$, y el segundo, la relación estudiante-docente-saber y contexto $(27,27 \%)$.

El siguiente texto obtenido en la primera aplicación del cuestionario ilustra como criterio para el desarrollo de la argumentación elementos que hacen referencia solo a los alumnos:

Pregunta 4: Expresa dos criterios que debe tener en cuenta un docente para desarrollar la argumentación en clase de ciencias.

Docente: Desarrollo intelectual de los niños y niñas, capacidad de comprensión y análisis.

Una posible interpretación del texto anterior permite identificar que para el docente el aprendizaje depende del desarrollo del individuo. Perspectiva que se aleja de la teoría sociocultural, por la que el aprendizaje es un proceso de interacción social que requiere, para su desarrollo, interacciones dialógicas entre docente, estudiantes, saber y contexto escolar.

En la segunda aplicación del cuestionario surgió, como criterio para el desarrollo de la argumentación, la relación entre estudiante-docente-saber y contexto:

Pregunta 4: Expresa dos criterios que debe tener en cuenta...

Docente: Crear una clase con las condiciones adecuadas, siempre con el apoyo del docente, identificar afirmaciones y datos; trabajar en equipo con preguntas orientadoras; que el estudiante aprenda a escuchar, hablar y justificar sus conclusiones; aprender a elegir entre diferentes opciones.

Del texto anterior, podemos decir tres cosas: a) la función que asume el docente es la de servir de apoyo para la construcción de la clase; visión en la que las relaciones de poder, si bien existirán en el interior del aula, no son tan notorias, pues la interacción se caracteriza por las relaciones simétricas alumno-docente (modelo sistémico, para Contreras, 1990, citado por Levin, Ramos y Adúriz-Bravo, 2008), y porque ellos son copartícipes de la creación de ese escenario de interacción (Wolfe y Alexander, 2008); b) el trabajo en equipo, elemento clave para desarrollar la argumentación en clase de ciencias (Osborne, Erduran y Simon, 2004; Jiménez-Aleixandre, 2010), y c) la elección de diferentes opciones: recordemos que uno de los procesos básicos para la evolución de la ciencia es la elección de los modelos teóricos que se proponen, optando por el que mejor explica, en su momento, el fenómeno (Giere, 1999). En la respuesta hay una intencionalidad concreta, y es que los estudiantes aprendan a "elegir la mejor opción", intencionalidad cercana al procedimiento aplicado en la construcción del conocimiento científico. 


\section{Las actividades para el desarrollo de la argumentación}

La pregunta cinco del cuestionario indagó específicamente este elemento. En el cuestionario inicial, las respuestas ofrecidas por el grupo de docentes manifiestan tanto para las actividades individuales como para las actividades colectivas el mismo porcentaje de aceptación. En la segunda aplicación del cuestionario, hubo un cambio importante de perspectiva, pues los docentes dieron mayor relevancia a las actividades grupales como mecanismo para el desarrollo de la argumentación, con un 90 frente al $10 \%$ obtenido para las actividades individuales.

En la siguiente respuesta obtenida en la primera aplicación del cuestionario vemos que la actividad es individual y asume además que los estudiantes son receptores de estímulos externos y de información:

Pregunta 5: Si uno de los objetivos de tus clases es promover la argumentación de tus alumnos, escribe dos actividades que pueden favorecerla. Justifica el porqué de cada una de ellas.

Docente: Hacer experimentos. Con plantas, seres vivos, etc. Y que los niños vayan observando el comportamiento a través de cada etapa del proceso.

En la segunda aplicación del cuestionario identificamos cambios con respecto al tipo de actividad grupal:

Pregunta 5: Si uno de los objetivos de tus clases es promover la argumentación...

Docente: Lo primordial es el trabajo grupal con un moderador que recoja los conceptos, los escriba, que los alumnos interactúen, que haya participación

Es notoria la intención de realizar pequeños grupos, en los cuales se expongan ideas que luego serán socializadas ante la clase. Esta gestión del aula permite promover en los alumnos, además de la capacidad de síntesis, otras capacidades como la escucha, la crítica de saberes y opiniones, así como la construcción de consensos y comprensiones conjuntas.

\section{Aspecto estructural}

En la siguiente tabla se muestran los resultados obtenidos con relación al aspecto estructural, después de la primera y segunda aplicación del cuestionario.

Tabla 5.

Resultados obtenidos para el aspecto estructural en la aplicación inicial y final del cuestionario

\begin{tabular}{|l|c|c|c|c|}
\hline \multirow{3}{*}{ Aspecto } & Categoría & \multirow{2}{*}{ Subcategoría } & \multicolumn{2}{|c|}{ Resultados cuestionario } \\
\cline { 3 - 5 } & & & Inicial (100\%) & Final (100\%) \\
\hline \multirow{3}{*}{ Estructural } & \multirow{3}{*}{ Nivel de los argumentos ${ }^{2}$ construidos por los docentes } & 1 & 33,11 & 9,37 \\
\cline { 3 - 5 } & & 2 & 41,67 & 53,13 \\
\cline { 3 - 5 } & & 4 & 13,90 & 31,25 \\
\cline { 3 - 5 } & & 5 & 8,33 & 6,25 \\
\hline
\end{tabular}

2. Son cinco los niveles propuestos por Erduran, Simon y Osborne (2004). En el nivel 1 se encuentran los argumentos que solo presentan afirmaciones. En el nivel 2 están los argumentos formados por conclusiones y al menos datos, justificaciones o apoyos. En el nivel 3 se hallan los argumentos cuya estructura la constituyen las conclusiones con al menos datos, justificaciones o apoyos y alguna refutación débil. Aquí se planteó una variación al nivel, pues ubicamos en el nivel 3 los argumentos que presentasen conclusiones, datos y justificaciones. En el nivel 4 se tienen los argumentos que muestran claramente una conclusión y una refutación. En el nivel 5 se sitúan los argumentos caracterizados por tener conclusiones y más de una refutación. 
Las preguntas 1, 2, 5 y 6 se plantearon para identificar, en el grupo de docentes, la estructura de sus argumentos. En la primera aplicación del cuestionario (tabla 5) identificamos que el mayor porcentaje de los textos construidos por los docentes se ubicó en el nivel argumentativo 2, con un 41,67\%, seguido por el nivel 1, con el 33,11\%, luego tenemos el nivel 3, con el 13,8\%, y por último el nivel 4, con un $8,33 \%$. En la segunda aplicación del cuestionario, los resultados variaron en todos los niveles. Se observó, en primer lugar, que tanto el nivel 2 como el 3 aumentaron el porcentaje de los textos ubicados en ellos, 53,13\% para el nivel 2 y $31,25 \%$ para el nivel 3 . Los otros dos niveles disminuyeron el porcentaje de los textos, el 1 pasó al 9,37 y el 4 al 6,25\%.

Del cuestionario inicial, una de las respuestas dadas por los docentes ante la pregunta 5 y ubicada en el nivel argumentativo 1 fue la siguiente:

Pregunta 5: Si uno de los objetivos de tus clases es promover la argumentación de tus alumnos, escribe dos actividades que pueden favorecerla. Justifica el porqué de cada una de ellas.

Docente:

a) Uso de analogías. Los argumentos por analogías, indicando que los dos ejemplos son semejantes en muchos aspectos generales y específicos.

b) Estudio de caso. Al estudiante se le presenta el resumen de un artículo de una revista científica.

En la respuesta, la docente afirma que el estudio de casos o la analogía es una actividad para favorecer el desarrollo de la argumentación, sin presentar el porqué de su utilización. Argumento que se ubica, según la escala argumentativa de Erduran, Simon y Osborne, (2004), en el nivel 1. Un texto donde se evidencia solo la conclusión o afirmación, como respuesta a una demanda o argumentos de reconvención o una reclamación frente a la demanda, está dentro del nivel argumentativo 1.

La respuesta siguiente, identificada en la segunda aplicación del cuestionario, es un ejemplo de un texto argumentativo del nivel 3:

Pregunta 6: Si uno de los objetivos de tus clases es promover la argumentación...

Docente:

a) Partir de situaciones cotidianas. Proponer un tema contextualizado que sea del interés de los educandos, ya que a través de él se pueden explorar cada uno de los elementos de la argumentación, enseñándoles a tomar posturas, a escuchar al otro, a convencer y a persuadir analizando los cambios en las concepciones.

b) Crear un ambiente favorable, así los alumnos saben que van a argumentar; mostrar una actitud siempre muy favorable; disponer de ayudas didácticas: guías, experimentos, láminas y lecturas, entre otras; que aprendan a trabajar en equipo, especialmente eligiendo entre varias opciones.

En el texto se pueden identificar claramente datos, conclusiones y justificaciones (figuras 1a y $1 b)$ :

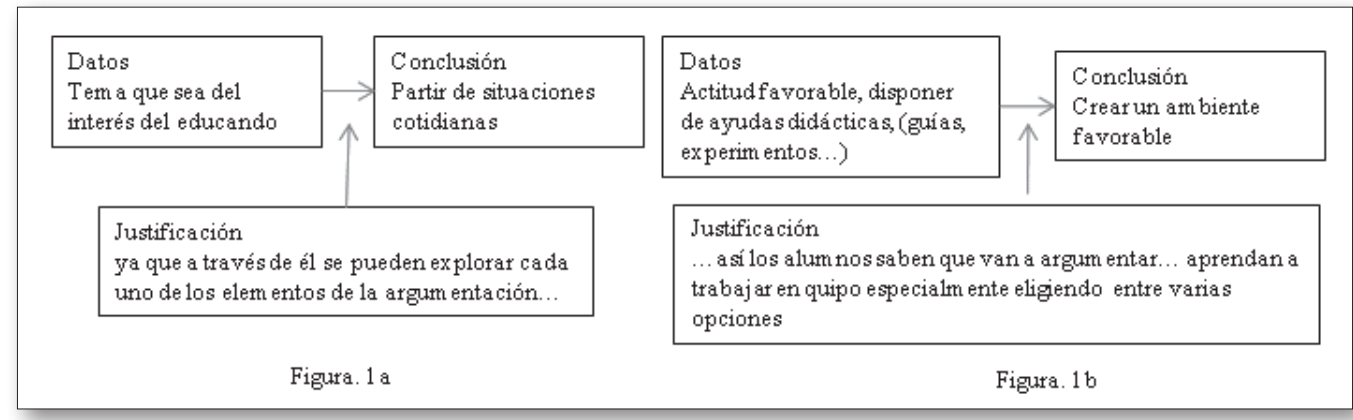

Figuras 1a y 1b. Estructura argumentativa de la respuesta de la docente para los ítems a y b de la pregunta 6 del cuestionario aplicado 


\section{Conclusiones e implicaciones}

Las conclusiones que se presentan a continuación se enfocan a resaltar dos elementos. El primero, las concepciones de los docentes sobre la argumentación y su desarrollo en el aula. El segundo, la estructura de los argumentos construidos por los docentes que participaron en el proceso.

- En relación con las concepciones, en los tres aspectos indagados se obtuvieron cambios significativos:

- En el aspecto epistemológico, los docentes reconocieron de manera explícita la relación entre la argumentación y la construcción de la ciencia, que pasan de posturas epistemológicas tradicionales de la construcción de la ciencia (caracterizadas por el empirismo y racionalismo) a posturas en las cuales la ciencia es considerada como una actividad humana mediada por procesos de interacción dialógica y afectados por elementos de orden social y cultural.

- En el aspecto conceptual se detectaron tres elementos de cambio. El primero, relacionado con el fortalecimiento de la perspectiva funcional de la argumentación, es decir, considerar la argumentación como un proceso social y dialógico, donde interesan, además de los argumentos, los sujetos y el contexto en el cual suceden los debates. El segundo elemento, el cambio en la intencionalidad de la argumentación, que pasa de ser una acción para informar a una acción para persuadir y convencer. El tercer elemento de cambio, el surgimiento de una nueva concepción, afín con la perspectiva funcional, en la que se visualiza la argumentación como una herramienta útil para evaluar los aprendizajes de los estudiantes.

- En el aspecto didáctico destacan dos cambios en los docentes. El primero, el convencimiento que el trabajo en pequeños grupos y la interacción entre los sujetos que lo integran son una plataforma de potenciación y desarrollo de la argumentación en el aula. El segundo, el reconocimiento de la relación estudiante-docente-saber-contexto como criterio necesario para el desarrollo de procesos argumentativos. Criterio vinculado a la concepción funcional de la argumentación en la cual, como lo manifestamos anteriormente, argumentar exige valorar tanto a los sujetos, sus saberes y opiniones, como el contexto en el cual tienen lugar las interacciones dialógicas.

En relación con el aspecto estructural, el análisis de las respuestas escritas de los profesores desde el punto de vista de su estructura argumentativa permitió detectar un aumento en el uso de datos y apoyos en las conclusiones, aunque no un cambio significativo. Este resultado es el esperado, puesto que durante el proceso no se trabajó ni reflexionó de manera específica la manera de argumentar.

En definitiva, los hallazgos obtenidos permiten afirmar que el proceso de reflexión crítica es un mecanismo de soporte que facilita que el profesorado participe de manera más activa en la regulación de sus maneras de pensar en torno a la argumentación y a su implementación en la clase de ciencias.

\section{RECONOCIMIENTOS}

Este estudio se desarrolló dentro de los proyectos EDU 2009-13890-C02-02 y EDU 2012-38022C02-02, financiado por la Dirección General de Investigación, Ministerio de Educación y Ciencia. También nuestro reconocimiento a los docentes que de manera voluntaria hicieron parte de este estudio, y a la Universidad de Caldas por su vital apoyo para culminar esta investigación. 


\section{BIBLIOGRAFÍA}

Angulo, F. (2002). Aprender a enseñar ciencias: análisis de una propuesta para la formación inicial del profesorado de secundaria basada en la metacognición. Tesis de grado obtenido no publicada. Barcelona: Universidad Autónoma de Barcelona, Bellaterra.

Benarroch, A. y Marín, N. (2011). Relaciones entre creencias sobre enseñanza, aprendizaje y conocimiento de ciencias. Enseñanza de las ciencias, 29(2), pp. 289-304.

Bravo, B.; Puig, B y Jiménez-Aleixandre, M. (2009). Componentes en el uso de pruebas de argumentación. Educación Quimica, 20(2), pp. 137-142.

Caicedo, H. (1999). Ideas actuales sobre la enseñanza de la ciencia. Bogotá: Pontificia Universidad Javeriana.

Campaner, G. y De Longhi, A. (2005, octubre). Enseñar a argumentar. Un aporte a la Didáctica de las ciencias. Ponencia presentada en el Tercer Encuentro de Investigadores en Didáctica de la Biología. Ciudad Autónoma de Buenos Aires, Argentina.

Dolz, J. (1995). Escribir textos argumentativos para mejorar su comprensión. Comunicación, lenguaje y educación, 26, pp. 65-79.

Driver, R.; Newton, P. y Osborne, J. (1998). Establishing the Norms of Scientific Argumentation in Classrooms. Science Education, 84(3), pp. 287-312.

Duschl, R. (1998). La valoración de argumentaciones y explicaciones: promover estrategias de retroalimentación. Enseñanza de las ciencias, 16(1), pp. 3-20.

Erduran, S. y Villamanan, R. (2009). Cool Argument: Engineering Students' Written Arguments about Thermodynamics in the Context of the Peltier Effect in Refrigeration. Educación Quimica, 20(2), pp. 119-125.

Erduran, S.; Simon, S y Osborne, J. (2004). TAPping into Argumentation: Developments in the Application of Toulmin's Argument Pattern for Studying Science Discourse. Wiley InterScience. Disponible en: <www.interscience.wiley.com>. (Última consulta: 1 de febrero de 2009).

Fernández, I.; Gil, D. y Carrascosa, J. (2002). Visiones deformadas de la ciencia transmitidas por la enseñanza. Enseñanza de las ciencias, 20(3), pp. 477-488.

Fernández, M.; Tuset, A.; Pérez, R. y Leyva, A. (2009). Concepciones de los maestros sobre la enseñanza y el aprendizaje y sus prácticas educativas en clases de ciencias naturales. Enseñanza de las ciencias, 27(2), pp. 287-298.

Giere, R. (1988). Explaining Science. A Cognitive Approach. Chicago: University of Chicago Press.

Giere, R. (1999). Science without Laws. Chicago: University of Chicago Press.

GiL, F. y Rico, L. (2003). Concepciones y creencias del profesorado de secundaria sobre enseñanza y aprendizaje de las matemáticas. Enseñanza de las ciencias, 21(1), 27-47.

Gunstone, R.; Slattery, M.; Bair, J. y Northrield, J. (1993). A case study exploration of development in preservice science teachers. Science Education, 77(1), pp. 47-73.

Henao, B. y STipcich, M. (2008). Educación en ciencias y argumentación: la perspectiva de Toulmin como posible respuesta a las demandas y desafíos contemporáneos para la enseñanza de las Ciencias Experimentales. Revista Electrónica de Enseñanza de las Ciencias, 7(1), pp. 47-62.

Disponible en: <http://reec.uvigo.es/volumenes/volumen7/ART3_Vol7_N1.pdf>. (Última consulta: 26 de septiembre de 2009).

Hernández, R. (2000, mayo). Aproximación a un estado del arte de la investigación en la enseñanza de las ciencias en Colombia. Ponencia presentada en el seminario La investigación educativa y pedagógica en Colombia. Balance de una década. Bogotá, Colombia.

Hoffman, M. (2008). Reflective argumentation. Disponible en: <http://www.spp.gatech.edu/faculty/ workingpapers/wp44.pdf>. (Última consulta: 13 de octubre de 2009). 
IzQuierdo, M. (2006). Por una enseñanza de las ciencias fundamentada en valores humanos. Revista Mexicana de investigación educativa, 11(30), pp. 867-882.

Jiménez- Aleixandre, M. (2010). 10 ideas clave. Competencias en argumentación y uso de pruebas. Barcelona: Graó.

Jiménez-Aleixandre, M. y Erduran, S. (2008). Argumentation Science Education: An Overview. En M. P. Jiménez-Aleixandre y S. Erduran (eds). Argumentation in science education. Perspectives from classroom-based research. Dordrecht: Springer, pp. 3-27

Jiménez Aleixandre, M. y Díaz, B. (2003). Discurso de aula y argumentación en la clase de ciencias: cuestiones teóricas y metodológicas. Enseñanza de las ciencias, 21(3), pp. 359-370.

Kaufman, M. y Fumagalli, L. (2000). Enseñar Ciencia Naturales. Reflexiones y propuestas didácticas. Barcelona: Paidós.

LARrain, S. (2007). Condiciones retóricas y semióticas en el proceso de auto-argumentación reflexiva. Tesis de grado obtenido no publicada. Pontificia Universidad Católica de Chile, Chile.

Lemke, J. (1997). Aprender a hablar ciencia. Barcelona: Ediciones Paidós.

Levin, L.; Ramos, A. y Adúriz-Bravo, A. (2008). Procesos de enseñanza y modelos de comunicación en clase de ciencias naturales. Disponible en: <http://www.pedagogica.edu.co/revistas/ojs/index.php/ TED/article/viewArticle/147>. (Última consulta: 14 de octubre de 2010).

Ley General de Educación (1994). Bogotá: Ministerio de Educación Nacional.

Llinares, S. (1996). Conocimiento profesional del profesor de matemáticas: conocimiento, creencias y contexto en relación con la noción de función. En J. Ponte et al. (coords.). Desenvolvimento profissional des professores de matemática. Què formação? Serção de Educação Matemática. Lisboa: Sociedad Portuguesa de Ciencias de Educação, pp. 47-82.

Marcelo, C. (2005). La investigación sobre el conocimiento de los profesores y el proceso de aprender a enseñar. En G. Perafán y A. Adúriz-Bravo (comps.). Pensamiento y Conocimiento de los profesores. Debate y perspectivas internacionales. Bogotá D.C.: Universidad Pedagógica Nacional, pp. 45-60.

Márquez, C. (2005). Aprender ciencias a través del lenguaje. Educar, 33, pp. 27-38.

Mellado, V.; Ruiz, M. y Blanco, L. (1997). Aprender a enseñar ciencias experimentales en la formación inicial del profesorado. Bordón, 49(3), pp. 275-288.

Moreno, M. y Ascárate, G. (2003). Concepciones de los profesores universitarios de matemáticas acerca de la enseñanza de las ecuaciones químicas diferenciales. Enseñanza de las ciencias, 21(1), pp. $27-47$.

Osborne, J.; Erduran, S. y Simon, S. (2004). Ideas, evidence and argument in science (IDEAS). Inservice Training Pack, Resource Pack and Video. Londres: Nuffield Foundation.

Porlán, R.; Rivero, A. y Martín Del P. (1997). Conocimiento profesional y epistemología de los profesores, I: Teoría, métodos e instrumentos. Enseñanza de las ciencias, 15(2), pp. 155-171.

Porlán, R.; Rivero, A. y Martín Del P. (1998). Conocimiento profesional y epistemología de los profesores, II: Estudios empíricos y conclusiones. Enseñanza de las ciencias, 16(2), pp. 271-288.

Revel, Ch.; Couló, A.; Sibel, E.; Furman, M.; Iglesia, P. y Adúriz-Bravo, A. (2005). Estudios sobre la enseñanza de la argumentación. Enseñanza de las ciencias. Número extra.

Reyes, L.; Enrique, L. y Perafán, G. (1997). Acciones y creencias, tesoro oculto del educador. Santafé de Bogotá: Universidad Pedagógica Nacional.

Sanmartí, N. (1997). Enseñar a elaborar textos científicos en clase de ciencias. Alambique, 12, pp. 51-61.

Sanmartí, N. (coord.) (2003). Aprendre ciències tot aprenent a escriure ciència. Barcelona: Edicions 62.

SARdÀ, A. y Sanmartí, N. (2000). Enseñar a argumentar científicamente: un reto de las clases de ciencias. Enseñanza de las ciencias, 18(3), pp. 405-422. 
Schôn, D. (1998). El profesional reflexivo: cómo piensan los profesionales cuando actúan. Barcelona: Paidós.

Schwarz, Ch.; Reiser, B.; Davis, E.; Kenyon, L.; Achér, A.; Fortus, D. et al. (2009). Developing a Learning Progression for Scientific Modeling: Making Scientific Modeling Accessible and Meaningful for Learners. Journal of Research In Science Teaching, 46(6), pp. 632-654.

Simon, S. H.; Erduran, S. y Osborne, J. (2006). Learning to Teach: Research and development in the science classroom. International Journal of Science Education, 28(2), pp. 235-260.

Sutton, C. (2003). Los profesores de ciencias como profesores de lenguaje. Enseñanza de las ciencias, 21(1), pp. 21-25.

Sutton, C. (1997). Ideas sobre la ciencia e ideas sobre el lenguaje. Alambique, 12, pp. 8-32.

Toulmin, S. (1969). The Uses of Argument. Cambridge. England: Cambridge University Press.

Von Aufschnaiter, C.; Erduran, S.; Osborne y Simon, S. (2008). Arguing to Learn and Learning to Argue: Case Studies of How Students' Argumentation Relates to Their Scientific Knowledge. Journal of Research In Science Teaching, 45(1), pp. 101-131.

Wolfe, S. y Alexander, R. (2008). Argumentation and dialogic teaching: alternative pedagogies for a changing world. Disponible en: <http://www.beyondcurrenthorizons.org.uk/wp-content/uploads/ ch3_final_wolfealexander_argumentationalternativepedagogies_20081218.pdf>. (Última consulta: 11 de julio de 2010).

Zohar, A. y Nemet, F. (2002). Fostering Students' Knowledge and Argumentation Skills Through Dilemmas in Human Genetics. Journal of Research in Science Teaching, 39(1), pp. 35-62. 


\title{
Teachers' change of conceptions on argumenta- tion and its development in science class
}

\author{
Francisco Javier Ruiz Ortega \\ Universidad de Caldas, Manizales, Colombia \\ francisco.ruiz@ucaldas.edu.co \\ Conxita Márquez Bargalló \\ Universidad Autónoma de Barcelona \\ Conxita.Marquez@uab.es \\ Óscar Eugenio Tamayo Alzate \\ Universidad de Caldas, Manizales, Colombia \\ oscar.tamayo@ucaldas.edu.co
}

The research entitled Characterization and evolution of the teaching models of argumentation in science class in primary school worked with a group of 5 primary teachers who belonged to a school named "Fe y Alegría" in the city of Manizales, Colombia and taught lessons to children aged between 9 and 10 years old. Two out of those five teachers were selected for the case study.

This research had three goals: (a) To characterize and understand the changes in the approach to argumentation and in the argumentative capacity of the sample population, (b) to identify the teaching models of argumentation in the science classroom of the teachers involved in the process of critical reflection, (c) to understand the evolution of teaching models of argumentation identified in the teachers sampled. The theoretical framework highlights four aspects: (a) argumentation as scientific competence; (b) the Toulmin's theory of argumentation and an adaptation and enrichment of this theory in the field of science education (c) the importance for science education to involve teachers in critical reflection processes and, finally, (d) the integration of the approach and performance of the teachers to identify and characterize teaching models of argumentation in science.

Methodologically, this research is situated within the qualitative paradigm and it highlighted the complementary elements of ethnography, action research and case study designs for in-depth understanding of the phenomena. The information was gathered from various sources: a) a questionnaire consisting of open questions and applied before and after the critical reflection process (a process that lasted seven months), b) the teachers' lesson planning, c) video and audio classes recordings, interviews and critical reflection meetings conducted with teachers.

A strict monitoring of the process, along with the content and discourse analysis made to such information, allowed to characterize the teachers' approach and their performance which, after their integration, constituted the basis for understanding the evolution of three teaching models of argumentation in science.

The results showed how criticism toward aspects of order such as: (a) epistemological, specifically about the traditional perspective of building science; (b) didactical, specifically referring to the authoritarian role of the teacher, manifested in the dogmatic conception of science introduced in the classroom and in the authoritarian communicative interactions between teachers and students and (c) conceptual, particularly the fact of assuming argumentation as an activity to give information based on data and claims; all of them fostering changes in the teachers' performance that helped to promote the gradual evolution of teaching models of argumentation.

Finally, this research suggests some implications that contribute to its strengthening and also to the start of new research proposals, such as the study of argumentative actions of students in the science classroom in primary education or evaluation of the contributions of textbooks to argumentative processes. 